\title{
TRANSFORM AND INVERSE TRANSFORM EXPANSIONS FOR SINGULAR SELF-ADJOINT DIFFERENTIAL OPERATORS ${ }^{1}$
}

\author{
$\mathrm{BY}$ \\ N. LLEVINSON
}

1. Coddington [2] has obtained the expansion theorem for singular selfadjoint ordinary linear differential operators with the most general type of boundary condition that can be associated with a self-adjoint problem. His proofs are elegant and are based on the Green's function method he had earlier introduced and on the boundary conditions he had shown to be appropriate to self-adjoint problems [1].

Here the inverse transform expansion will be proved. The method used here combines the procedure used by the author [4, also 3, pp. 261-272] with the boundary conditions as given by Coddington in [2] for the transform expansion. In the course of proving the inverse transform expansion theorem, the transform expansion result due to Coddington will also be proved.

Let $L$ be the differential operator

$$
L=p_{0}\left(\frac{d}{d t}\right)^{n}+p_{1}\left(\frac{d}{d t}\right)^{n-1}+\cdots+p_{n}
$$

where the $p_{j}$ are complex-valued functions of $t$ with $n-j$ continuous derivatives on the open interval $(a, b)$. The cases $a=-\infty, b=\infty$, or both are allowed. Let $p_{0}(t) \neq 0$ on $(a, b)$, and let $L$ be identical with its adjoint defined by

$$
(-1)^{n}\left(\frac{d}{d t}\right)^{n}\left(\bar{p}_{0} \cdot \quad\right)+(-1)^{n-1}\left(\frac{d}{d t}\right)^{n-1}\left(\bar{p}_{1} \cdot \quad\right)+\cdots+\left(\bar{p}_{n} \cdot \quad\right) .
$$

Let $\mathfrak{H C}$ denote the set of all measurable functions which have square integrable magnitude on $(a, b)$. Let $\mathfrak{D}$ denote the subset of $\mathfrak{H C}$ consisting of those elements which are of class $C_{n-1}$ on $(a, b)$ with $(n-1)^{\text {st }}$ derivative absolutely continuous on every closed subinterval of $(a, b)$ and such that any $\phi \epsilon \mathcal{D}$ satisfies $L \phi \in \mathfrak{H C}$.

Let $x$ and $y$ be in D, and let $a<t_{1}<t_{2}<b$. Then Green's formula is

$$
\int_{t_{1}}^{t_{2}}(\bar{y} L x-x \overline{L y}) d t=[x y]\left(t_{2}\right)-[x y]\left(t_{1}\right)
$$

where

$$
[x y](t)=\sum_{m=1}^{n} \sum_{j+k=m-1}(-1)^{j} x^{(k)}(t)\left(p_{n-m} \bar{y}\right)^{(j)}(t) .
$$

Received July 29, 1957.

1 This paper was written in the course of research sponsored by the Office of Naval Research. 
From Green's formula it follows that

$$
[x y](a)=\lim _{t_{1} \rightarrow a+0}[x y]\left(t_{1}\right), \quad[x y](b)=\lim _{t_{2} \rightarrow b-0}[x y]\left(t_{2}\right)
$$

exist for $x, y$ in $D$. Let $\langle x y\rangle=[x y](b)-[x y](a)$.

It will be assumed that the equations $L x=i x$ and $L x=-i x$ each have exactly $\omega$ linearly independent solutions which are in $D$ where $0 \leqq \omega \leqq n$. Let $\phi_{1}, \phi_{2}, \cdots, \phi_{\omega}$ be an orthonormal set of solutions of $L x=i x$ in $D$, and $\psi_{1}, \cdots, \psi_{\omega}$ a set of $L x=-i x$ in $D$. Iet $U=\left(u_{j k}\right), j, k=1, \cdots, \omega$, be a unitary matrix, and let

$$
v_{j}=\phi_{j}-\sum_{k=1}^{\omega} u_{j k} \psi_{k}, \quad j=1, \cdots, \omega .
$$

Let $\mathfrak{D}_{U}$ denote the subset of $\mathfrak{D}$ such that for any $x \in \mathscr{D}_{U}$

$$
\left\langle x v_{j}\right\rangle=0
$$$$
j=1, \cdots, \omega .
$$

The equations (1.3) are in a sense a set of $\omega$ boundary conditions. (It is shown in Coddington [1] that to any self-adjoint problem associated with $L$ on $(a, b)$ there corresponds a unique $U$ and conversely. This fact is not required in our proofs.)

Let $l$ be a complex valued parameter, and let $\chi_{k}(t, l), k=1, \cdots, n$, be the solutions of $L x=l x$ which at some $c, a<c<b$, satisfy

$$
\chi_{k}^{(j-1)}(c, l)=\delta_{j k}, \quad j, k=1, \cdots, n,
$$

where $\delta_{j j}=1$ and $\delta_{j k}=0, j \neq k$. Clearly the $\chi_{k}$ are independent solutions of $L x=l x$.

The hermitian matrix $\rho=\left(\rho_{j k}(\sigma)\right)$ is said to be nondecreasing if $\rho(\sigma)-\rho(\lambda)$ is positive semidefinite for $\sigma>\lambda$.

Theorem 1. Corresponding to the conditions (1.3), there exists on $(-\infty, \infty)$ a unique hermitian, nondecreasing matrix, $\rho(\sigma)$, with elements of bounded variation on every finite interval. If $f(t) \in \mathfrak{L}^{2}(a, b)$, then

$$
g_{j}(\sigma)=\int_{a}^{b} f(t) \bar{\chi}_{j}(t, \sigma) d t
$$

exists (in the sense of convergence in the mean in the norm of (1.6)), and

$$
f(t)=\int_{-\infty}^{\infty} \sum_{j, k=1}^{n} \chi_{j}(t, \sigma) g_{k}(\sigma) d \rho_{j k}(\sigma)
$$

(where again the integral converges in the mean). Moreover if

is denoted by

$$
\int_{-\infty}^{\infty} \sum_{j, k=1}^{n} g_{j}(\sigma) \bar{g}_{k}(\sigma) d \rho_{j k}(\sigma)
$$

$$
\int_{-\infty}^{\infty} g \bar{g} d \rho
$$


then

$$
\int_{a}^{b}|f|^{2} d t=\int_{-\infty}^{\infty} g \bar{g} d \rho .
$$

Further for any given $l$, $g l \neq 0$, there exists a unique $F(t)$ in $\mathfrak{D}_{U}$ such that

$$
(L-l) F=f
$$

Moreover

$$
F(t)=\int_{-\infty}^{\infty} \sum_{j, k=1}^{n} \frac{\chi_{j}(t, \sigma) g_{k}(\sigma) d \rho_{j k}(\sigma)}{\sigma-l}=\int_{-\infty}^{\infty} \frac{\chi g}{\sigma-l} d \rho
$$

Theorem 2 (Inverse transform). Let the vector, $g_{j}(\sigma), j=1, \cdots, n$, be measurable with respect to $\rho(\sigma)$, and let

$$
\int_{-\infty}^{\infty} g \bar{g} d \rho<\infty
$$

Then there exists a unique $f \in \mathfrak{L}^{2}(a, b)$ given by (1.5). Moreover $g_{j}$ is then given by (1.4).

Theorem 3. Let the two functions $F^{(1)}$ and $F^{(2)}$ be in $D_{U}$. Then

$$
\int_{a}^{b}\left(\bar{F}^{(2)} L F^{(1)}-F^{(1)} \overline{L F^{(2)}}\right) d t=0
$$

2. The singular problem is handled as in [4] by first using a closed interval $\delta=[\alpha, \beta]$ interior to $(a, b)$ and then letting $\delta \rightarrow(a, b)$. However now the boundary conditions associated with the nonsingular problem on $\delta$ must be based on (1.3) exactly as was done in [2]. It will be assumed that $\alpha$ is near enough to $a$ and $\beta$ to $b$ so that $\alpha \leqq c \leqq \beta$. The Gram-Schmidt process is now used to obtain the orthonormal functions $\phi_{1 \delta}, \cdots, \phi_{\omega \delta}$ on $\delta$ from $\phi_{1}, \cdots, \phi_{\omega}$. Similarly $\psi_{1 \delta}, \cdots, \psi_{\omega \delta}$ are obtained from $\psi_{1}, \cdots, \psi_{\omega}$. Here

$$
\phi_{j \delta}=\sum_{1}^{\omega} a_{j k}(\delta) \phi_{k}, \quad \psi_{j \delta}=\sum_{1}^{\omega} b_{j k}(\delta) \psi_{k},
$$

where $A(\delta)=\left(a_{j k}(\delta)\right)$ and $B(\delta)=\left(b_{j k}(\delta)\right)$ are constant matrices and

$$
A(\delta) \rightarrow E, \quad B(\delta) \rightarrow E, \quad \text { as } \quad \delta \rightarrow(a, b),
$$

where $E$ is the unit matrix of $\omega$ rows and columns. Let $\phi_{\omega+1 \delta}, \cdots, \phi_{n \delta}$ be chosen so that $\phi_{1 \delta}, \cdots, \phi_{n \delta}$ form an orthonormal set of solutions of $L x=i x$ on $\delta$. Let $\psi_{1 \delta}, \cdots, \psi_{n \delta}$ be a similar set for $L x=-i x$ on $\delta$. Let $U$ be the matrix introduced in (1.2), and let $U(\delta)$ be the unitary matrix with elements

$$
\begin{array}{lrl}
u_{j k}(\delta) & =u_{j k}, & j, k=1, \cdots, \omega, \\
u_{j j}(\delta)=1, & j=\omega+1, \cdots, n, \\
u_{j k}(\delta)=0, & \text { all other } j, k \leqq n .
\end{array}
$$


Let

$$
\begin{array}{lrl}
v_{j \delta}=\phi_{j \delta}-\sum_{k=1}^{\omega} u_{j k} \psi_{j \delta}, & j=1, \cdots, \omega . \\
v_{j \delta}=\phi_{j \delta}-\psi_{j \delta}, & j=\omega+1, \cdots, n .
\end{array}
$$

These equations can also be written as

$$
v_{j \delta}=\phi_{j \delta}-\sum_{k=1}^{n} u_{j k}(\delta) \psi_{j \delta} .
$$

The nonsingular boundary value problem on $\alpha \leqq t \leqq \beta$

$$
L x=l x, \quad\left\langle x v_{j \delta}\right\rangle_{\delta}=0,
$$$$
j=1, \cdots, n,
$$

where $\left\langle x v_{j \delta}\right\rangle_{\delta}=\left[x v_{j \delta}\right](\beta)-\left[x v_{j \delta}\right](\alpha)$, is self-adjoint as is shown in [1].

Thus (2.3) has characteristic values and characteristic functions and an expansion theorem. As in [4, or 3] this expansion theorem can be expressed in terms of the matrix $\rho_{\delta}$ which has as its elements step functions. For any $f$ of $\mathfrak{L}^{2}(\delta)$ if

$$
g_{j}(\sigma)=\int_{\alpha}^{\beta} f(t) \bar{\chi}_{j}(t, \sigma) d t
$$

$$
j=1, \cdots, n
$$

then by the standard expansion theorem on $[\alpha, \beta]$

$$
f(t)=\int_{-\infty}^{\infty} \sum \chi_{\jmath}(t, \sigma) g_{k}(\sigma) d \rho_{j k \delta}(\sigma),
$$

and

$$
\int_{\alpha}^{\beta}|f(t)|^{2} d t=\int_{-\infty}^{\infty} g \bar{g} d \rho_{\delta} .
$$

As is shown in [4, or 3, Chapter 10], the $\rho_{\delta}(\sigma)$ are uniformly of bounded variation on any given closed interval as $\delta \rightarrow(a, b)$, and hence by the Helly selection theorem there is a sequence $\delta_{j}, j=1,2, \cdots$, such that $\delta_{j} \rightarrow(a, b)$ and $\rho_{\delta_{j}}$ converges as $j \rightarrow \infty$. Denote the limit matrix by $\rho(\sigma)$. (Later it will be shown that $\rho$ is unique, and hence it will follow that at any two points of continuity $\lambda$ and $\sigma$ of $\rho$

$$
\rho_{\delta}(\sigma)-\rho_{\delta}(\lambda) \rightarrow \rho(\sigma)-\rho(\lambda) \text { as } \delta \rightarrow(a, b) .
$$

But for now $\rho(\sigma)$ is defined by the use of the sequence $\delta_{j}$.)

As was shown in [4, pp. 305-307, or 3, Chapter 10], once the existence of $\rho(\sigma)$ is established, it follows easily from the Parseval equality in the nonsingular case that (1.4), (1.5), and (1.7) hold. These results will be used here. Also required is the following lemma proved much as in [4, p. 307; or 3, Chapter 10].

Lemma 2.1 Let $G_{j}(\sigma), j=1, \cdots, n$, be measurable with respect to $\rho$, and let

$$
\int_{-\infty}^{\infty} G \bar{G} d \rho<\infty
$$


Let

$$
P_{A}(t)=\int_{-A}^{A} \sum \chi_{j}(t, \sigma) G_{k}(\sigma) d \rho_{j k}(\sigma)
$$

Then l.i.m. $\cdot_{A \rightarrow \infty} P_{A}(t)=P(t)$ exists, and

$$
\int_{a}^{b}|P(t)|^{2} d t \leqq \int_{-\infty}^{\infty} G \bar{G} d \rho
$$

3. To complete the proof of Theorem 1, $\rho$ must be proved unique, and the existence of a unique $F$ in $\mathfrak{D}_{U}$ satisfying (1.8) and (1.9) must be established.

Let $f(t)$ be $\mathscr{L}^{2}(a, b)$ and vanish outside of some finite closed subinterval of $(a, b)$. Assume $\delta=[\alpha, \beta]$ is taken so that outside of $[\alpha, \beta], f(t)$ vanishes. Let $g$ be defined as in (1.4). Then by the expansion theorem for the nonsingular problem

$$
f_{A \delta}(t)=\int_{-A}^{A} \sum \chi_{j} g_{k} d \rho_{j k \delta}(\sigma)
$$

converges in the mean to $f$ over $\delta$ as $A \rightarrow \infty$. Let $g l \neq 0$, and let

$$
F_{A \delta}(t)=\int_{-A}^{A} \sum \frac{x_{j} g_{k}}{(\sigma-l)} d \rho_{j k \delta} .
$$

The integrals in (3.1) or (3.2) are simply finite sums of several eigenfunctions associated with (2.3). Since each eigenfunction satisfies the boundary conditions of (2.3), it follows that

$$
\left\langle F_{A \delta} v_{j \delta}\right\rangle_{\delta}=0, \quad j=1, \cdots, n .
$$

Also by differentiating (3.2) there follows

$$
(L-l) F_{A \delta}=f_{A \delta} .
$$

Now by Green's formula

$$
\left\langle F_{A \delta} v_{j \delta}\right\rangle_{\delta}=\int_{\alpha}^{\beta}\left(\bar{v}_{j \delta} L F_{A \delta}-F_{A \delta} \overline{L v}_{j \delta}\right) d t .
$$

By the definition of $\phi_{j \delta}, \psi_{j \delta}$ it follows that for $j=1,2, \cdots, \omega$

$$
\int_{a}^{b}\left|\phi_{j}-\phi_{j \delta}\right|^{2} d t+\int_{a}^{b}\left|\psi_{j}-\psi_{j \delta}\right|^{2} d t \rightarrow 0 \quad \text { as } \delta \rightarrow(a, b) .
$$

From the definition of $v_{j \delta}$ this implies for $j=1,2, \cdots, \omega$

$$
\int_{a}^{b}\left|v_{j}-v_{j \delta}\right|^{2} d t \rightarrow 0 \quad \text { as } \delta \rightarrow(a, b)
$$

Now by (2.6)

$$
\int_{-\infty}^{\infty} g \bar{g} d \rho_{\delta}=\int_{\alpha}^{\beta}|f|^{2} d t,
$$


and from the orthogonality of the characteristic functions

$$
\int_{\alpha}^{\beta}\left|F_{A \delta}\right|^{2} d t=\int_{-A}^{A} \frac{g \bar{g}}{|\sigma-l|^{2}} d \rho_{\delta} \leqq \frac{1}{|g l|^{2}} \int_{a}^{b}|f|^{2} d t
$$

and similarly

$$
\int_{\alpha}^{\beta}\left|f_{A \delta}\right|^{2} d t \leqq \int_{a}^{b}|f|^{2} d t
$$

By (3.4) the right side of (3.5) can be written as

$$
\int_{\alpha}^{\beta}\left(\bar{v}_{j \delta}\left(l F_{A \delta}+f_{A \delta}\right)-F_{A \delta} \overline{L v}_{j \delta}\right) d t .
$$

For $j \leqq \omega, L v_{j \delta}$ is a fixed linear combination of $\phi_{j \delta}$ and $\psi_{j \delta}, j \leqq \omega$. Hence by (3.6) for $j \leqq \omega$

$$
\int_{a}^{b}\left|L v_{j}-L v_{j \delta}\right|^{2} d t \rightarrow 0 \quad \text { as } \quad \delta \rightarrow(a, b) .
$$

If $\delta \rightarrow(a, b)$ through the sequence $\delta_{j}$ described below (2.6), and if

$$
F_{A}(t)=\int_{-A}^{A} \sum \frac{\chi_{j} g_{k}}{\sigma-l} d \rho_{j k}
$$

then

$$
F_{A \delta}(t)-F_{A}(t) \rightarrow 0
$$

uniformly over any closed subinterval of $(a, b)$. Similar results hold for $f_{A}(t)$, where

$$
f_{A}(t)=\int_{-A}^{A} \sum \chi_{j} g_{k} d \rho_{j k}
$$

From (3.11) follows by differentiating

$$
(L-l) F_{A}=f_{A} .
$$

Let $a<a_{1}<b_{1}<b$. Then (3.9) can be written as

$$
\int_{\alpha}^{\beta}=\int_{\alpha}^{a_{1}}+\int_{a_{1}}^{b_{1}}+\int_{b_{1}}^{\beta}
$$

From (3.7), (3.10), and (3.12) as $\delta \rightarrow(a, b)$ through the sequence $\delta_{j}$

$$
\int_{a_{1}}^{b_{1}}\left(\bar{v}_{j \delta}\left(l F_{A \delta}+f_{A \delta}\right)-F_{A \delta} \overline{L v}_{j \delta}\right) d t \rightarrow \int_{a_{1}}^{b_{1}}\left(\bar{v}_{j}\left(l F_{A}+f_{A}\right)-F_{A} \overline{L v}_{j}\right) d t .
$$

Also by using (3.8)

$$
\int_{\alpha}^{a_{1}}\left|v_{j \delta} F_{A \delta}\right| d t \leqq\left(\int_{a}^{a_{1}}\left|v_{j \delta}\right|^{2} d t\right)^{1 / 2}\left(\int_{a}^{b}|f|^{2} d t\right)^{1 / 2} /|\mathfrak{g} l| .
$$


By using (3.7) the first term on the right tends to

$$
\left(\int_{a}^{a_{1}}\left|v_{j}\right|^{2} d t\right)^{1 / 2}
$$

as $\delta \rightarrow(a, b)$, and this can be made as small as desired by choosing $a_{1}$ near enough to $a$. Similar results hold for the terms $\int f_{A \delta} \bar{v}_{j \delta} d t$ and $\int F_{A \delta} \overline{L v}_{j \delta} d t$ and for the interval $\left(b_{1}, \beta\right)$.

From (3.8) follows easily, by first using a fixed closed subinterval of $(a, b)$, that

$$
\int_{a}^{b}\left|F_{A}\right|^{2} d t \leqq \int_{a}^{b}|f|^{2} d t /|\mathfrak{g} l|^{2}
$$

Using this, (3.7), and (3.10) shows the right side of (3.15) can be made arbitrarily close to

$$
\int_{a}^{b}\left(\bar{v}_{j}\left(l F_{A}+f_{A}\right)-F_{A} \overline{L v}_{j}\right) d t=\left\langle F_{A} v_{j}\right\rangle
$$

by choice of $a_{1}$ and $b_{1}$. All these remarks together show that (3.5) tends to (3.16) as $\delta \rightarrow(a, b)$. By (3.3), (3.5) is zero, and hence so is (3.16). Thus

$$
\left\langle F_{A} v_{j}\right\rangle=0 \text {, }
$$$$
j=1,2, \cdots, \omega \text {. }
$$

For any $C>0$

$$
\int_{-C}^{C} g \bar{g} d \rho_{\delta} \leqq \int_{a}^{b}|f|^{2} d t
$$

By letting $\delta \rightarrow(a, b)$ through the sequence $\delta_{j}$

Next letting $C \rightarrow \infty$

$$
\int_{-C}^{C} g \bar{g} d \rho \leqq \int_{a}^{b}|f|^{2} d t
$$

$$
\int_{-\infty}^{\infty} g \bar{g} d \rho \leqq \int_{a}^{b}|f|^{2} d t
$$

When $A \rightarrow \infty$, it is the case that l.i.m. A $_{\rightarrow \infty} f_{A}=f$ by (1.5). If we let $A \rightarrow \infty$, it follows from (3.11) and Lemma 2.1 that there exists $F(t)$ in $\mathfrak{L}^{2}(a, b)$ such that l.i.m. $._{A \rightarrow \infty} F_{A}=F$. In (3.16) these lead readily to the fact that as $t \rightarrow \infty$ the left side of (3.16) converges to

$$
\int_{a}^{b}\left(\bar{v}_{j}(l F+f)-F \bar{L}_{j}\right) d t=0
$$

since (3.16) was shown to be zero.

If $F_{A}$ is represented as a solution of $(L-l) F_{A}=f_{A}$ by means of the variation of constants formula much as in [4], then it follows easily that $F$ also is representable by this formula and hence $F$ is in $D$, and moreover

$$
(I,-l) F=f .
$$

Using (3.20) in (3.19) gives

$$
\left\langle F v_{j}\right\rangle=0, \quad j=1, \cdots, \omega .
$$


Hence $F^{\prime}$ is in $\mathfrak{D}_{U}$ and is given by

$$
F(t)=\underset{A \rightarrow \infty}{\lim . m} \int_{-A}^{A} \sum \chi_{j} g_{k} d \rho_{j k}(\sigma) /(\sigma-l) .
$$

Finally the restriction that $f$ vanishes outside of some closed subinterval of $(a, b)$ can be removed. Indeed any $f$ of $\mathfrak{L}^{2}(a, b)$ can be approximated in $\mathfrak{L}^{2}(a, b)$ by a sequence of restricted functions $f_{s}, s=1,2, \cdots$, which converges to $f$ in $\mathfrak{L}^{2}(a, b)$. Let the $g_{j}$ and $F$ associated with $f_{s}$ be denoted by $g_{s j}$ and $F_{s}$. Then an argument analogous to that used in proceeding from $f_{A}$ to $f$ above now can be used to deal with $f_{8}, g_{s j}$, and $F_{s}$ as $s \rightarrow \infty$, and the result is the validity of (3.20), (3.21), and (3.22) for any $f$ of $\mathcal{L}^{2}(a, b)$. This leaves only the demonstration of the uniqueness of $F$ and $\rho$ to complete the proof of Theorem 1 .

It will be shown next that if $l=i$ then $F$ as given by (3.22) is unique in the sense that

$$
(L-i) F^{\prime}=0, \quad\left\langle F v_{j}\right\rangle=0, \quad j=1, \cdots, \omega,
$$

has $F=0$ as its only solution in $D_{U}$.

To prove this, note that

$$
\left\langle\phi_{j} \phi_{k}\right\rangle=\int_{a}^{b}\left(\bar{\phi}_{k} L \phi_{j}-\phi_{j} \overline{L \phi}_{k}\right) d t=2 i \int_{a}^{b} \phi_{j} \bar{\phi}_{k} d t
$$

Hence

$$
\left\langle\phi_{j} \phi_{j}\right\rangle=2 i ; \quad\left\langle\phi_{j} \phi_{k}\right\rangle=0, \quad j \neq k .
$$

Similarly

$$
\left\langle\phi_{j} \psi_{k}\right\rangle=0 ; \quad\left\langle\psi_{j} \psi_{j}\right\rangle=-2 i ; \quad\left\langle\psi_{j} \psi_{k}\right\rangle=0, \quad j \neq k .
$$

From this follows easily

$$
\left\langle\phi_{j} v_{j}\right\rangle=2 i ; \quad\left\langle\phi_{k} v_{j}\right\rangle=0, \quad k \neq j .
$$

Now any solution of $(L-i) F=0$ which is $\mathscr{L}^{2}(a, b)$ must be of the form

$$
F=\sum_{1}^{\omega} C_{k} \phi_{k}
$$

where the $C_{k}$ are constants. By using $\left\langle F v_{j}\right\rangle=0$ and (3.26) it follows that all $C_{k}$ are zero and hence $F=0$. A similar argument applies if $L-i$ is replaced by $L+i$ in (3.23).

Now suppose for some $l_{0}, g l_{0} \neq 0$, it is known that $\left(L-l_{0}\right) F=0$ has $F=0$ as its only solution in $D_{U}$. Then it will be proved that this is true for $(L-l) F^{\prime}=0$ inside the circle $\left|l-l_{0}\right|<\left|g l_{0}\right|$. Indeed for some such $l$ let $(L-l) H=0$, where $H$ is not identically zero and is in $D_{U}$. Iet

$$
G_{j}=\int_{a}^{b} H \bar{\chi}_{j} d t
$$

'Then

$$
\int_{a}^{b}|H|^{2} d t=\int_{-\infty}^{\infty} G \bar{G} d \rho .
$$


Clearly

Hence by (3.22)

$$
\left(L-l_{0}\right) H=\left(l-l_{0}\right) H, \quad\left\langle H v_{j}\right\rangle=0 .
$$

By Lemma 2.1

$$
H(t)=\int_{-\infty}^{\infty} \frac{l-l_{0}}{\sigma-l_{0}} \sum \chi_{j} G_{k} d \rho_{j k}
$$

$$
\int_{a}^{b}|H|^{2} d t \leqq \int_{-\infty}^{\infty}\left|\frac{l-l_{0}}{\sigma-l_{0}}\right|^{2} G \bar{G} d \rho .
$$

But

$$
\left|\frac{l-l_{0}}{\sigma-l_{0}}\right| \leqq\left|\frac{l-l_{0}}{g l_{0}}\right|<1 .
$$

Hence using this in (3.28) and using (3.27) yield

$$
\int_{a}^{b}|H|^{2} d t<\int_{a}^{b}|H|^{2} d t
$$

which proves that indeed $H=0$. Since uniqueness was shown for $l_{0}= \pm i$, the above argument extends this to the upper and lower halves of the $l$-plane.

There remains now only to show the uniqueness of $\rho$. Suppose there is some $\hat{\rho}$ with the properties of Theorem I which have been demonstrated for $\rho$. (Note that $\hat{\rho}$ need not necessarily arise from any limiting process with a sequence of $\left.\rho_{\delta}\right)$. Let $g l>0$, and let $f$ be $\mathscr{L}^{2}(a, b)$. Then the unique solution of $(L-l) F=f$ in $D_{U}$ is given by (3.22) with $\rho$ replaced by $\hat{\rho}$. Subtracting gives

$$
0=\underset{A \rightarrow \infty}{\lim .} \int_{-A}^{A} \sum \frac{\chi_{j} g_{k}}{\sigma-l} d(\rho-\hat{\rho})_{j k}
$$

Let

$$
\Gamma_{j}(\sigma, \tau)=\int_{c}^{\tau} \bar{\chi}_{j}(t, \sigma) d t
$$

Integrating (3.29) with respect to $t$ from $c$ to $\tau$ gives

$$
\int_{-\infty}^{\infty} \sum \bar{\Gamma}_{j}(\sigma, \tau) g_{k}(\sigma) d(\rho-\hat{\rho})_{j k} /(\sigma-l)=0
$$

As in $[4,(4.10)]$ this leads readily to the identity of $\rho$ and $\hat{\rho}$. The argument is analogous to that below (4.9) of the next section.

4. In proving Theorem 2 it suffices to restrict the $g_{j}(\sigma)$ to be continuous and zero outside of some closed interval since these are dense in $\mathfrak{L}^{2}(\rho)$. Denote such a continuous vector vanishing outside of a finite interval by $G_{j}(\sigma)$, $j=1, \cdots, n$. Then obviously one can define

$$
f(t)=\int_{-\infty}^{\infty} \sum \chi_{j}(t, \sigma) G_{k}(\sigma) d \rho_{j k}(\sigma)
$$

and for $g l \neq 0$

$$
F(t)=\int_{-\infty}^{\infty} \sum \chi_{j} G_{k} d \rho_{j k} /(\sigma-l)
$$


exists, and

$$
(L-l) F=f
$$

If

$$
f_{\delta}=\int_{-\infty}^{\infty} \sum \chi_{j} G_{k} d \rho_{j k \delta}
$$

and

$$
F_{\delta}=\int_{-\infty}^{\infty} \sum \chi_{j} G_{k} d \rho_{j k \delta} /(\sigma-l)
$$

then

and

$$
(I, l) H_{\delta}^{\prime}=f_{\delta}
$$

$$
\left\langle F_{\delta} v_{j \delta}\right\rangle_{\delta}=0,
$$$$
j=1, \cdots, n,
$$

since $F_{\delta}$ as given by (4.5) is simply the sum of a finite number of characteristic functions associated with the nonsingular self-adjoint boundary problem on $\delta=[\alpha, \beta]$. By using the argument of (3.1)-(3.17) it follows that (4.6) implies

$$
\left\langle F v_{j}\right\rangle=0,
$$$$
j=1, \cdots, \omega \text {. }
$$

However the uniform bounds on

$$
\int_{\alpha}^{\beta}\left|f_{\delta}\right|^{2} d t, \quad \int_{\alpha}^{\beta}\left|F_{\delta}\right|^{2} d t
$$

which are required must now be derived differently. By using the Parseval equality, (4.4) and (4.5), in terms of the orthogonal characteristic functions, give

Since as $\delta \rightarrow(a, b)$

$$
\int_{\alpha}^{\beta}\left|f_{\delta}\right|^{2} d t=\int_{-\infty}^{\infty} G \bar{G} d \rho_{\delta}
$$

$$
\int_{-\infty}^{\infty} G \bar{G} d \rho_{\delta} \rightarrow \int_{-\infty}^{\infty} G \bar{G} d \rho
$$

it follows that if $\delta$ is near enough to $(a, b)$,

Similarly

$$
\int_{\alpha}^{\beta}\left|f_{\delta}\right|^{2} d t \leqq 1+\int_{-\infty}^{\infty} G \bar{G} d \rho
$$

$$
\int_{\alpha}^{\beta}\left|F_{\delta}\right|^{2} d t \leqq 1+\int_{-\infty}^{\infty} G \bar{G} d \rho /(g l)^{2}
$$

From Lemma 2.1 follows

$$
\begin{aligned}
& \int_{a}^{b}|f|^{2} d t \leqq \int_{-\infty}^{\infty} G \bar{G} d \rho \\
& \int_{a}^{b}|F|^{2} d t \leqq \int_{-\infty}^{\infty} G \bar{G} d \rho /(\mathfrak{g} l)^{2} .
\end{aligned}
$$

With these four inequalities the argument leading to (3.17) now leads to (4.7). 
Thus $F$ is in $\mathfrak{D}_{U}$ and is the solution of (4.3). Since $f$ is in $\mathfrak{L}^{2}(a, b)$, it follows from Theorem 1 that if the transform of $f$ is defined by

then

$$
g_{j}(\sigma)=\int_{a}^{b} f(t) \bar{\chi}_{j}(t, \sigma) d t
$$

$$
F(t)=\underset{A \rightarrow \infty}{\operatorname{li} . m} . \int_{-A}^{\Lambda} \sum \chi_{j} g_{k} d \rho_{j k} /(\sigma-l) .
$$

Integrating (4.2) and (4.8) from $c$ to $\tau$ and subtracting gives

$$
\int_{-\infty}^{\infty} \sum \bar{\Gamma}_{j}(\sigma, \tau)\left(G_{k}-g_{k}\right) d \rho_{j k} /(\sigma-l)=0,
$$

where $\Gamma_{j}$ is given by (3.30). Since $\Gamma_{j}$ and $\left(G_{k}-g_{k}\right)$ are both $\mathfrak{L}^{2}(\rho),(4.9)$ converges absolutely by the Schwartz inequality. Writing (4.9) for $\vec{l}$, and letting $l=u+i v$ and subtracting the two cases gives

$$
\int_{-\infty}^{\infty} \frac{v}{(\sigma-u)^{2}+v^{2}} \sum \bar{\Gamma}_{\jmath}\left(G_{k}-g_{k}\right) d \rho_{j k}=0
$$

Integrating with respect to $u$ from $\sigma_{1}$ to $\sigma_{2}$, where $\sigma_{1}$ and $\sigma_{2}$ are any two points of continuity of $\rho$, and then letting $v \rightarrow 0$ yields

$$
\int_{\sigma_{1}}^{\sigma_{2}} \sum \bar{\Gamma}_{j}(\sigma, \tau)\left(G_{k}-g_{k}\right) d \rho_{j k}=0
$$

Differentiating with respect to $\tau$ and replacing $\tau$ by $t$ gives

$$
\int_{\sigma_{1}}^{\sigma_{2}} \sum \chi_{j}(t, \sigma)\left(G_{k}-g_{k}\right) d \rho_{j k}=0
$$

Differentiating $(s-1)$ times with respect to $t$ and then putting $t=c$, and using $\chi_{j}^{(s-1)}(c, \sigma)=\delta_{j s}$ gives

$$
\int_{\sigma_{1}}^{\sigma_{2}} \sum_{k=1}^{n}\left(G_{k}-g_{k}\right) d \rho_{s k}=0, \quad s=1, \cdots, n .
$$

Replacing $s$ by $j$ and using the arbitrary character of $\sigma_{1}$ and $\sigma_{2}$ leads to

$$
\int_{-A}^{A} h_{j}(\sigma) \sum_{k=1}^{n}\left(G_{k}-g_{k}\right) d \rho_{j k}=0, \quad j=1, \cdots, n,
$$

for any continuous $h_{j}(\sigma)$ and any $A$. Summing on $j$, we have

$$
\int_{-A}^{A} \sum h_{j}\left(G_{k}-g_{k}\right) d \rho_{j k}=0
$$

Since $G-g$ is in $\mathscr{L}^{2}(\rho)$, the arbitrary character of $h$ implies that the left side can be made arbitrarily close to

$$
\int_{-A}^{A}(G-g)(\overline{G-g}) d \rho
$$


and thus the above must be zero. Since this holds for all $A$,

$$
\int_{-\infty}^{\infty}(G-g)(\overline{G-g}) d \rho=0
$$

which proves Theorem 2 .

5. Here Theorem 3 will be proved. From (1.7) follows easily that if $f^{(1)}$ and $f^{(2)}$ are in $\mathcal{L}^{2}(a, b)$ and have transforms $g^{(1)}$ and $g^{(2)}$ respectively, then

$$
\int_{a}^{b} f^{(1)} \bar{f}^{(2)} d t=\int_{-\infty}^{\infty} g^{(1)} \bar{g}^{(2)} d \rho .
$$

In terms of $F^{(1)}$ and $F^{(2)}$ of Theorem 3 , let $f^{(1)}$ and $f^{(2)}$ be defined by

$$
f^{(1)}=(L-i) F^{(1)}, \quad f^{(2)}=(L+i) F^{(2)} .
$$

Then if $g^{(1)}$ and $g^{(2)}$ are the transforms of $f^{(1)}$ and $f^{(2)}$, it follows from Theorems 1 and 2 that the transform of $F^{(1)}$ is $g^{(1)} /(\sigma+i)$ and of $F^{(2)}, \mathrm{g}^{(2)} /(\sigma-i)$. Thus

and so

$$
\begin{aligned}
& \int_{a}^{b} f^{(1)} \bar{F}^{(2)} d t=\int_{-\infty}^{\infty} g^{(1)} \bar{g}^{(2)} d \rho /(\sigma+i), \\
& \int_{a}^{b} F^{(1)} \bar{f}^{(2)} d t=\int_{-\infty}^{\infty} g^{(1)} \bar{g}^{(2)} d \rho /(\sigma+i),
\end{aligned}
$$

$$
\int_{a}^{b}\left(f^{(1)} \bar{F}^{(2)}-F^{(1)} \bar{f}^{(2)}\right) d t=0 .
$$

Using (5.2) with this yields

$$
\int_{a}^{b}\left(\bar{F}^{(2)} L F^{(1)}-F^{(1)} \overline{L F}^{(2)}\right) d t=0,
$$

which proves Theorem 3 .

\section{REFERENCES}

1. E. A. Coddington, The spectral representation of ordinary self-adjoint differential operators, Ann. of Math. (2), vol. 60 (1954), pp. 192-211.

2. - - On self-adjoint ordinary differential operators, Math. Scand., vol. 4 (1956), pp. 9-21.

3. E. A. Coddington and N. Levinson, Theory of ordinary differential equations, New York, 1955.

4. N. Levinson, The expansion theorem for singular self-adjoint linear differential operators, Ann. of Math. (2), vol. 59 (1954), pp. 300-315.

Massachusetts Institute of Technology

Cambridge, Massachusetts 